\title{
PROGRAMA PARA INCENTIVO DAS ATIVIDADES ACADÊMICAS DOS ESTUDANTES DE ENGENHARIA BIOQUÍMICA DA UNIVERSIDADE FEDERAL DO RIO GRANDE - FURG: 9 ANOS
}

Laís A. Silva - laisapasilva@gmail.com

Michele da Rosa Andrade Zimmermann de Souza - michrandrade@ gmail.com

Alexia Silva da Rosa - lekah8@gmail.com

Susan Hartwig Duarte - susanduarte@gmail.com

Beatriz Precipito Peres - precipitobeatriz@outlook.com

Elisangela Martha Radmann - emradmann@yahoo.com.br

Karoline Pereira Rodrigues - karool.pereira06@gmail.com

Ana P. Centeno da Rosa - ana.centeno@furg.br

Amanda Wermelinger Borges - amandawermelinger@outlook.com

Universidade Federal do Rio Grande - FURG

Av. Itália, s/n - Km 8 - Carreiros, Rio Grande - RS

96203-900 - Rio Grande - RS

Resumo: No curso de engenharia cerca de metade dos ingressantes evade ou são retidos nas séries iniciais. Diversos ingressantes em Engenharia Bioquímica (cerca de 80\% em 2019) têm como primeira opção no SiSU (Sistema de Seleção Unificada), outros cursos. Uma grande parcela dos ingressantes (aproximadamente 48\% em 2019) não se sentiam devidamente esclarecidos sobre o curso e possibilidades de atuação profissional. Esses estudantes necessitam de esclarecimentos sobre o curso no qual ingressaram e sobre a profissão. $O$ projeto PAIEB (Programa para Incentivo das Atividades Acadêmicas dos Estudantes de Engenharia Bioquímica) busca acolher, informar e motivar esses estudantes, através de oficinas e interação com estudantes do final do curso ou profissionais já formados. As ações realizadas incentivam o estudo, a persistência e a organização do tempo, contribuindo no combate à evasão e retenção de discentes nas séries iniciais do curso de Engenharia Bioquímica e para uma formação qualificada. Este trabalho tem por objetivo apresentar o projeto e discutir alguns de seus principais resultados dos anos de 2019-2020, principalmente a grande parcela de alunos que se sentiram beneficiados pelas atividades realizadas $(94,7 \%)$ e esclarecidos sobre o curso e a profissão (100\%). Neste trabalho também são abordadas as adaptações realizadas devido à pandemia de COVID-19, a importância do projeto e os principais resultados nesse período excepcional - a ampliação do público beneficiado e o importante elo de ligação à Universidade durante a suspensão das aulas. Por fim, o projeto está alinhado com as novas Diretrizes Curriculares Nacionais para o Curso de graduação em Engenharia.

Palavras-chave: Evasão; Motivação de ingressantes, Retenção. 


\section{INTRODUÇÃO}

Nas séries iniciais de Engenharia, cerca de 50\% dos ingressantes desistem do curso, sendo que esta evasão ocorre majoritariamente nos dois primeiros anos da graduação, quando são ofertadas disciplinas básicas de Matemática, Física e Química (MADEIRA et al., 2018). A evasão e a retenção ocorrem, muitas vezes, devido a dificuldades nas disciplinas e falta de conhecimento sobre o que realmente significa ser um Engenheiro (BRITO et al., 2017), entre outros fatores, sendo um dos mais complexos e multifatoriais problemas encontrados na educação superior. A evasão, principalmente, significa uma série de desperdícios sociais, acadêmicos e econômicos (FILHO et al., 2007).

Algumas das principais causas de evasão nos curso de Engenharia no Brasil relatadas na literatura são a falta de tempo para o estudo pela necessidade de trabalhar, desmotivação para o estudo em função de práticas pedagógicas ultrapassadas, falta de identificação do aluno com a área que está cursando, dificuldades de relacionamento com docentes do curso, dificuldades de aprendizado, baixo desempenho nas avaliações iniciais, dificuldade de adaptação ao ensino superior, o não atendimento das expectativas dos estudantes por parte do curso, entre outros (REIS et al., 2012).

Conhecendo essa problemática e aproveitando a experiência dos professores do grupo, há 9 anos vêm sendo desenvolvido o PAIEB - Programa para Incentivo das Atividades Acadêmicas dos Estudantes de Engenharia Bioquímica da FURG - através do qual são realizadas uma série de atividades para auxiliar no combate à evasão, retenção e a favor de uma formação profissional qualificada. O objetivo deste trabalho é apresentar esse programa e discutir alguns de seus resultados obtidos nos anos de 2019-2020.

\subsection{METODOLOGIA}

A equipe de trabalho do PAIEB é composta por 4 professores do curso de Engenharia Bioquímica, um bolsista do Programa de Bolsas de Ensino, Pesquisa, Extensão e Cultura da FURG (EPEC-FURG) e 4 bolsistas voluntários. A colaboração de outros voluntários é constantemente buscada no decorrer das atividades e a adesão de estudantes voluntários sempre ocorre com sucesso. $O$ projeto vem sendo realizado há 9 anos, sempre com modificações para melhorias. Este trabalho concentra-se nas atividades e resultados dos anos 2019-2020.

Antes do início de cada semestre letivo é sempre organizado pela equipe de trabalho um cronograma de atividades a serem realizadas; o projeto é divulgado durante as matrículas e durante a Acolhida aos Calouros, que acontece nas duas primeiras semanas letivas de cada semestre. $\mathrm{O}$ material para divulgação inclui folderes impressos, contato pessoal e por redes sociais (Facebook e Instagram). Quando o semestre letivo inicia é mantida a divulgação através de redes sociais e iniciada a divulgação pessoalmente nas turmas de calouros (público alvo do projeto) e nas turmas de terceiro semestre em diante, a fim de captar voluntários interessados em colaborar. Posteriormente é realizado um levantamento sobre a turma de ingressantes, através de um questionário com perguntas principalmente a respeito da faixa etária, cidade natal, residência, escola de origem, desempenho em disciplinas da área de exatas durante ensino médio, ano de conclusão do ensino médio, se Engenharia Bioquímica havia sido sua primeira opção no SiSU e sobre o esclarecimento em relação ao curso e à profissão de Engenheiro Bioquímico. Os resultados desse questionário são apresentados aos estudantes como um perfil de turma e, a partir dele, dúvidas são detectadas e atividades específicas planejadas. 
Uma das principais atividades realizadas no âmbito do projeto são os "Depoimentos de veteranos e egressos" relatando sua trajetória acadêmica, suas maneiras de ultrapassar as dificuldades em disciplinas, metodologias de estudos utilizadas e experiências extraclasse inclusive de estágios e intercâmbios, com objetivo de compartilhar suas experiências e mostrar oportunidades de formação aos calouros.

São também realizadas oficinas sobre assuntos importantes, mas não abordados em disciplinas do curso, como o uso de editores de texto, planilhas eletrônicas, elaboração e apresentação de trabalhos acadêmicos, preenchimento e importância do currículo lattes. Outras oficinas são oferecidas de acordo com a demanda detectada no questionário inicial ou sugeridas pelos estudantes, como as de Gerenciamento do Tempo e de Planejamento de Estudos. Para estas atividades colaboram psicólogos e pedagogos vinculados à Pró-Reitoria de Assuntos Estudantis da FURG. Na Oficina de Gerenciamento do Tempo é ressaltada a importância do equilíbrio entre atividades acadêmicas, sociais e físicas, por psicólogo com experiência em atendimento a acadêmicos, o que proporciona uma linguagem jovem e descontraída, importante na faixa etária da maioria dos ingressantes. Na Oficina de Planejamento de Estudos cada estudante constrói seu planejamento semanal de estudos e atividades, com o apoio de uma pedagoga, contando com tempo para descanso e lazer.

São também criados e acompanhados Grupos de Estudos para a discussão de temas inerentes ao curso de Engenharia Bioquímica, como produção de biogás, biodiesel e tratamento de resíduos. Esses grupos de estudo têm acolhido estudantes interessados em iniciação científica logo no início do curso, quando não há vagas suficientes para que todos sejam inseridos em projetos de pesquisa em laboratório.

Ao final de cada semestre são aplicados dois questionários: um de auto avaliação construído com apoio da equipe pedagógica que possibilita aos ingressantes refletir sobre atitudes e crescimento durante o semestre; e um questionário de avaliação do trabalho realizado, através o qual os ingressantes dão feedback sobre o projeto. Os resultados desses questionários são abordados nesse trabalho, comparando o início do $1^{\circ}$ semestre com o fim do $2^{\circ}$ semestre de 2019, ou seja, um ano de projeto. Em 2020, tudo começo normalmente, mas com o advento da pandemia de COVID-19, adaptações foram realizadas, e são apresentadas a seguir.

\section{RESULTADOS E DISCUSSÕES}

No ano de 2019 apenas 20,93\% (Tabela 1) dos estudantes optaram por Engenharia Bioquímica como primeira opção de curso no SiSU (Sistema de Seleção Unificada). Os demais estudantes tinham como primeira opção os cursos de Medicina, Farmácia e Biomedicina, entre outros relacionados à área da saúde. Esse resultado alertou para a necessidade de esclarecer logo no início do curso de forma bem simples "o que é estudado no curso de Engenharia Bioquímica", e "o que faz um Engenheiro Bioquímico", com base nas suas atribuições profissionais. A partir desse esclarecimento os estudantes podem decidir entre se manter no curso ou não. O objetivo principal é esclarecer, sem a pretensão de convencimento do estudante a permanecer no curso. Outras iniciativas como o site do curso (engbioquimica.furg.br), com o máximo de informação possível já são disponibilizadas, mas ainda assim, muitos desconhecem informações importantes que lhes evitaria ingressar em um curso de Engenharia, principalmente o grande volume de cálculos, inerente ao curso.

Quando perguntados "como o projeto os auxiliava em sua formação", diversos participantes destacaram ter aprendido a realizar edições e elaborações de textos e trabalhos acadêmicos, assim como a manipulação e a importância da ferramenta Excel para o curso de 
graduação. Outros tiveram esclarecidas suas dúvidas sobre o curso e à profissão. Alguns desses relatos, nas palavras dos próprios estudantes são transcritos:

"Aprendi a usar mais as ferramentas de escrita no editor de textos. Por exemplo, antes eu não sabia inserir fórmulas."

"O PAIEB me ajudou quanto ao Excel e a me planejar quanto à iniciação científica." "Ajudou na elaboração de textos."

"Não participei das atividades, mas de acordo com meus colegas, o projeto os ajudou muito."

“O PAIEB esclarece a maioria das dúvidas que temos no início da graduação."

"O projeto trouxe oficinas que me auxiliaram nos estudos."

"Me ensinou assuntos que não são abordados nas matérias da faculdade, mas são úteis para a formação."

"A oficina de Excel foi útil, pois esclareceu dúvidas sobre uma ferramenta importante para o curso."

"Aprendi muito com os cursos (oficinas) que foram aplicados."

"A oficina que participei ajudou a entender melhor o funcionamento da plataforma (lattes) apresentada."

"Me ajudou a aprender coisas novas, que contribuem tanto para meu currículo quanto para o desempenho em diversas matérias."

"Foi uma grande ajuda para a realização de trabalhos e listas de exercícios."

"Abriu minha mente para dúvidas antigas e de forma simples." 
"Me ajudou a desenvolver alguns trabalhos, me orientando para que sejam executados da melhor forma."

"O PAIEB me ajudou com conhecimentos que não ganhamos em aula."

Dos estudantes participantes, apenas 5,3\% declararam não se sentir auxiliados pelo projeto. Os dados sobre o $1^{\circ}$ semestre do ano de 2019 mostraram que 48,27\% dos ingressantes de Engenharia Bioquímica não se sentiam devidamente esclarecidos sobre o curso e possibilidades de atuação profissional. Esse número, entretanto, se modificou e caiu para 0 ao fim do $2^{\circ}$ semestre, quando $100 \%$ dos estudantes se sentiam devidamente esclarecidos sobre o curso de Engenharia Bioquímica e a profissão (Tabela 1). Esse crescimento é mais um indicador do sucesso do projeto, pois esse esclarecimento pode indiretamente contribuir no combate à evasão e retenção.

Ao fim do $2^{\circ}$ semestre letivo, 84,2\% dos estudantes decidiram permanecer na Engenharia Bioquímica. Esse número inclui estudantes que não tinham esse curso como primeira opção no SiSU (Tabela 1).

Tabela 1 - Esclarecimento quanto ao curso e profissão, primeira opção de curso no SISU e permanência na Engenharia Bioquímica.

\begin{tabular}{|c|c|c|}
\hline Pergunta & SIM & $\mathrm{NÃO}$ \\
\hline $\begin{array}{l}\text { Você se sente esclarecido sobre o que é o curso de Engenharia Bioquímica e a } \\
\text { profissão de Engenheiro Bioquímico? (Inicio do } 1^{\circ} \text { semestre) }\end{array}$ & $51,73 \%$ & $48,27 \%$ \\
\hline $\begin{array}{l}\text { Você se sente esclarecido sobre o que é o curso de Engenharia Bioquímica e } \\
\text { profissão de Engenheiro Bioquímico? (Fim do } 2^{\circ} \text { semestre) }\end{array}$ & $100 \%$ & $0 \%$ \\
\hline $\begin{array}{l}\text { Engenharia Bioquímica foi sua primeira opção de curso no SiSU? (Inicio do } 1^{\circ} \\
\text { semestre) }\end{array}$ & $20,93 \%$ & $79,07 \%$ \\
\hline Deseja permanecer no curso de Engenharia Bioquímica? (Fim do $2^{\circ}$ semestre) & $84,2 \%$ & $15,8 \%$ \\
\hline
\end{tabular}

Fonte: Autoras

A realização de atividades extraclasse com os quais os estudantes ingressantes pudessem se envolver, como a realização de grupos de estudo, oficinas e a interação com profissionais aumentaram o interesse e esclarecimento em relação ao curso. A apresentação aos estudantes em sala de aula, de grupos tutorial e de representação acadêmica, como o GTTEB (Grupo de Trabalho Tutorial em Engenharia Bioquímica) e o DAEB (Diretório Acadêmico da Engenharia Bioquímica), além de projetos de pesquisa (Iniciação científica) aumenta o interesse dos estudantes em atividades extraclasse, como demonstrado na Figura 1. 
Figura 1 - Intenção de envolvimento em atividades extraclasse.

Desejam realizar atividades extra curriculares nos próximos períodos

Não desejam participar de atividades extra curriculares nos próximos períodos

Já participam de atividades extra curriculares.

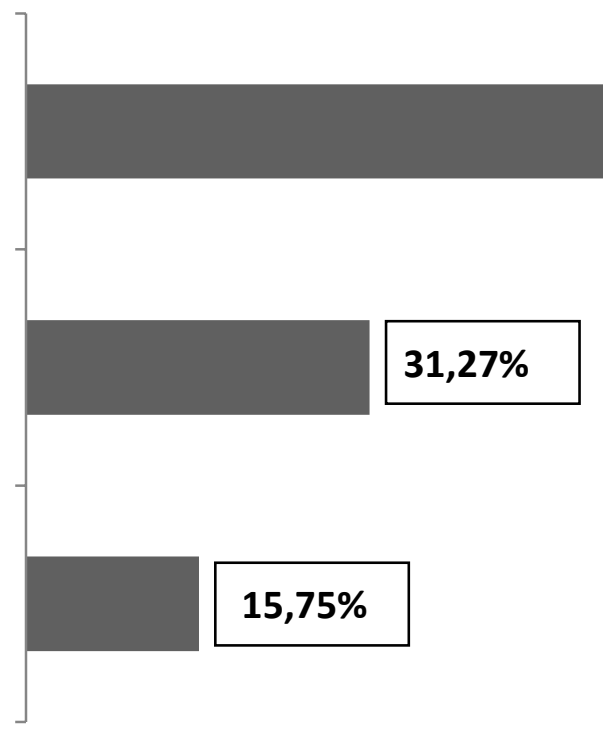

Fonte: Autoras

A Figura 2 apesenta a evasão no curso de Engenharia Bioquímica desde sua criação em 2010 até 2020. Juntamente com a criação do curso iniciou o projeto PAIEB, assim a comparação dos dados de evasão com e sem o trabalho realizado não é possível, então a eficácia das atividades realizadas deve ser medida por outros indicadores. Um deles é a avaliação positiva do projeto por parte dos participantes, obtida através dos questionários aplicados ao final de cada semestre. Neste, $94,7 \%$ dos estudantes participantes do projeto se declararam beneficiados pelas atividades realizadas.

Figura 2 - Evasão no curso de Engenharia Bioquímica da Universidade Federal do Rio

Grande (FURG).

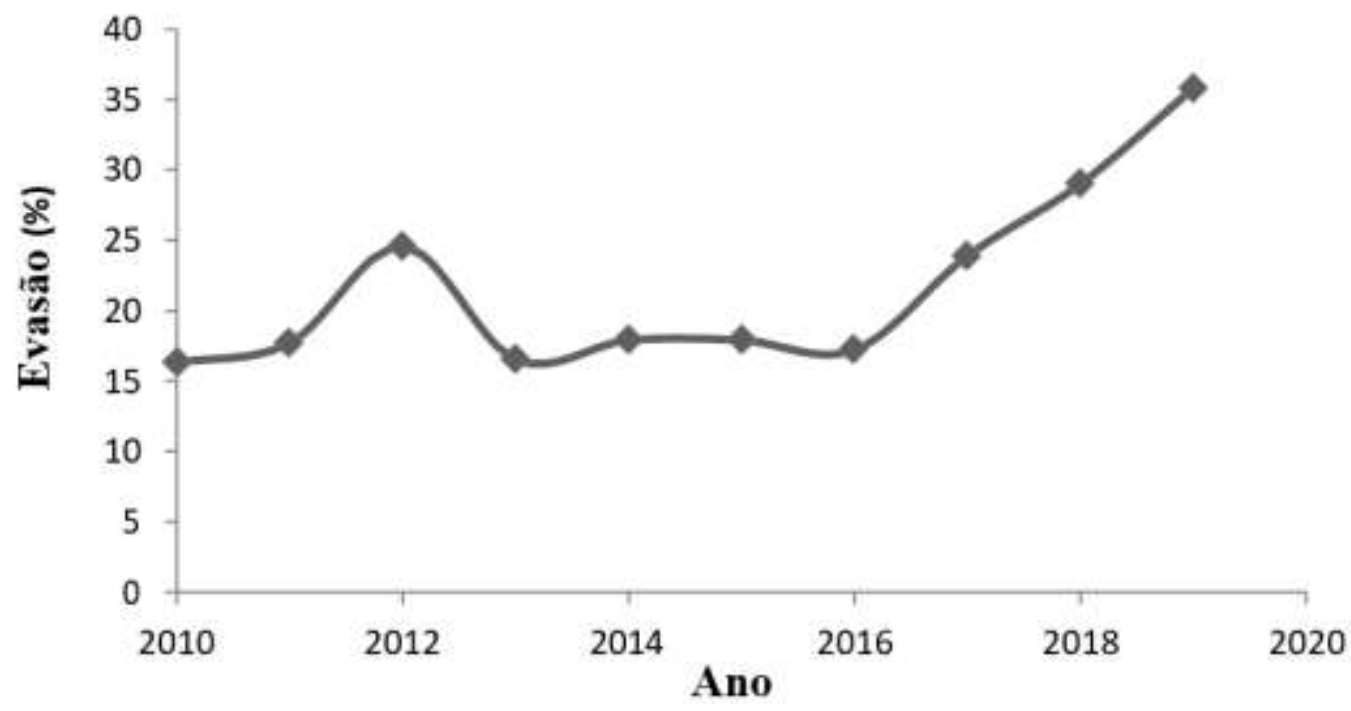

Fonte: Autoras 
Por fim a proximidade de alunos de séries iniciais com os de séries mais avançadas do curso, bem como com profissionais da área, pode gerar maior esclarecimento sobre "o que é ser Engenheiro Bioquímico" e assim, motivar calouros a persistir perante as dificuldades inerentes à Engenharia ou migrar para outro curso de maior interesse. Essa interação permite também ao ingressante vislumbrar algumas das diversas oportunidades de atuação profissional.

No início do primeiro semestre letivo de 2020, assim como ocorria desde o início da oferta do próprio curso de Engenharia Bioquímica, o projeto PAIEB iniciou suas atividades com a turma de ingressantes de maneira presencial, porém com o advento da pandemia de COVID19, foram necessárias adaptações para continuidade do trabalho. Nessa adaptação foi possível aumentar o alcance das atividades usando recursos on-line. De acordo com GLORIA e UTTAL (2020), nas últimas duas décadas houve uma nova compreensão de como ensinar online. O PAIEB incluiu-se nessa nova compreensão, aproveitando os recursos on-line para a continuidade dos trabalhos em meio à pandemia.

Nesse período o projeto foi um meio de auxiliar os estudantes a conhecerem mais sobre o curso no qual ingressaram, motivando-os a permanecer no curso com todas as dificuldades do ensino remoto emergencial que enfrentariam logo a seguir, entre outras inerentes ao momento de pandemia. A Figura 3 ilustra as páginas utilizadas pelo projeto para realização da divulgação das atividades on-line @PAIEBFURG (página na rede social Facebook) e @PAIEB.FURG (página na rede social Instagram).

Figura 3 - Páginas das redes sociais (Facebook e Instagram) utilizadas pelo PAIEB.

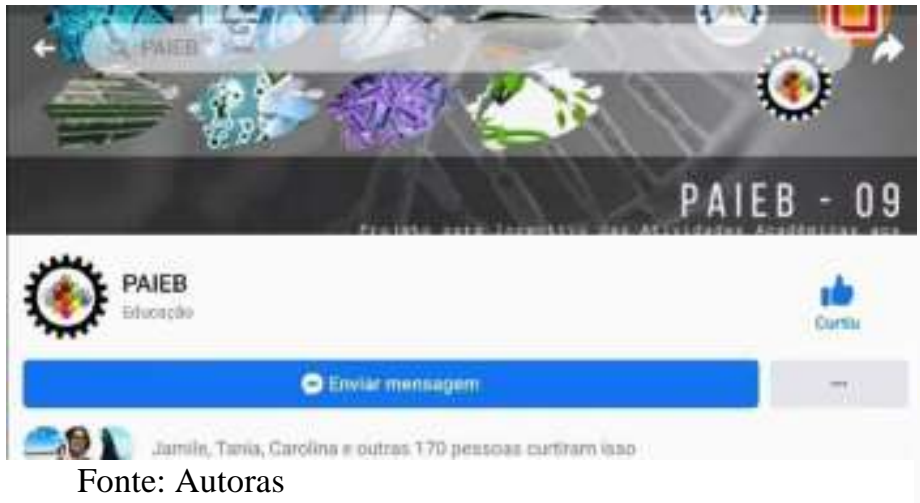

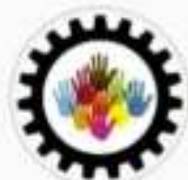

37

Publicaç... Sequidor... Seguindo

PAIEB

PAIEB - Programa de incentivo as atividades acadèmicas dos estudantes de Engenharia Bioquimica da FURG.

docs.google.com/forms/d/1_.MfZd-qoCJAQMo9Se.

Editar perfil

Utilizando plataformas online foram realizadas oficinas sobre Editores de Texto e Planilhas Eletrônicas, possibilitando a comunicação entre o ministrante e o espectador (síncronas). As páginas do projeto também foram utilizadas para realizar a aproximação de alunos veteranos e ingressantes através de vídeos onde os veteranos relatam experiências curriculares e extracurriculares no curso, como por exemplo, projetos de iniciação científica, estágios e intercâmbios. Assuntos no contexto da pandemia também foram abordados nas lives "Nutrição em tempos de pandemia" e "Saúde mental em meio à pandemia", abertas para comunidade interna e externa ao curso. As mudanças impostas pela pandemia de forma abrupta abriram novas possibilidades de atividades on-line no contexto do projeto, principalmente a expansão do público participante, que possivelmente se manterão. 


\section{CONSIDERAÇÕES FINAIS}

O problema de evasão e retenção na Engenharia é complexo, multifatorial e de difícil solução. Paralelamente, as novas DCNs (Diretrizes Curriculares Nacionais) do curso de graduação em Engenharia incluem o Acolhimento, que contempla esse cuidado com o estudante ingressante, objetivando tanto redução de evasão e retenção, quanto a formação profissional qualificada, recomendando atividades de nivelamento e atenção ao ingressante. Neste trabalho foram identificados possíveis fatores que podem levar à evasão e retenção no curso de Engenharia Bioquímica da FURG, principalmente o grande número de estudantes que não optaram por este curso como primeira opção no SiSU e o grande percentual de estudantes que desconhecem o próprio curso e a profissão de Engenheiro Bioquímico. Assim, é evidente a necessidade de ações que busquem o acolhimento, esclarecimento e envolvimento desses ingressantes com o curso. As atividades realizadas através do PAIEB vêm buscando esclarecer os ingressantes sobre o curso e aumentar o interesse e a motivação para os estudos, incentivando a persistência, a organização e promovendo a troca de experiências entre calouros e veteranos, assim contribuindo no combate à evasão e retenção de discentes nas séries iniciais e para uma formação qualificada. A experiência descrita neste trabalho pode ser útil a outras instituições, adaptadas a cada contexto.

\section{Agradecimentos}

Ao Programa EPEC - FURG pela bolsa concedida.

\section{REFERÊNCIAS}

BRITO, C. R et al. Engineering course specially designed to face retention issue. In: 45th SEFI Conference, 2017, Azores, Portugal (2017).

FILHO, R. L.L. et al. A evasão no ensino superior brasileiro Cadernos de Pesquisa, v. 37, n. 132, pág 642, set. 2007.

GLORIA, A.M. \& UTTAL, L. (2020). Conceptual Considerations in Moving from Face-toFace to Online Teaching. International Journal on E-Learning, Waynesville, USA, 23 Set. 2020. Association for the Advancement of Computing in Education (AACE), p139-159.

MADEIRA, V. et al., "A falta de preparação dos estudantes que ingressam nos cursos de engenharia no Brasil", 2018 IEEE Global Engineering Education Conference (EDUCON), 2018, Tenerife, Espanha (2018).

REIS, V. W; CUNHA, Paulo J.M; SPRITZER, Ilda M.P.A. Evasão no ensino superior de Engenharia no Brasil: um estudo de caso no CEFET/RJ. Vol 1, pág 3-4, setembro, 2012. 


\section{PROGRAM FOR ENCOURAGING THE ACADEMIC ACTIVITIES OF BIOCHEMICAL ENGINEERING STUDENTS AT THE FEDERAL UNIVERSITY OF RIO GRANDE - FURG: 9 YEARS}

Abstract: In the engineering course, about half of the new students evade or are retained in the first grades. Several new entrants in Biochemical Engineering (about 80\% in 2019) have other courses as their first option in SiSU (Unified Selection System). A large portion of new entrants (approximately 48\% in 2019) did not feel adequately informed about the course and possibilities for professional performance. These students study for clarifications about the course they entered and about the profession. The PAIEB project (Program for the Incentive of Academic Activities of Biochemical Engineering Students) seeks to receive, inform and motivate these students, through workshops and interaction with students at the end of the course or professionals already trained. The actions carried out encourage study, persistence and the organization of time, contributing to the fight against evasion and retention of students in the first series of the Biochemical Engineering course and for qualified training. This work aims to present the project and discuss some of its main results from the years 2019-2020, mainly the large portion of students who felt benefited by the activities carried out (94.7\%) and informed about the course and the profession (100\%). This work also addresses adaptations due to the COVID-19 pandemic, the importance of the project and the main results in this exceptional period - the expansion of the public benefited and the important link to the University during the suspension of classes. Finally, the project is aligned with the new National Curriculum Guidelines for the Engineering undergraduate course.

Keywords: Evasion; Motivation of freshmen; Action Strategy. 